\title{
Chemical Heterogeneous Surface and Its Application in Chemical Engineering
}

\author{
Yunshen Zhang
}

\section{Department of Petrochemical Engineering, Puyang Vocational and Technical College, puyang} Henan, P. R. China 457000

\section{Keywords: chemically heterogeneous surface; chemical industry; application}

\begin{abstract}
In the current study of chemistry, the unique properties of chemically heterogeneous surfaces in the nanoscale have attracted common attention of researchers. But how to apply this technology in actual chemical industry becomes common problem faced by researchers. This paper focuses on the advantages of chemistry application in the chemical heterogeneity surface to analyze, and discusses the effects of chemical non-uniform surfaces on molecular transport under confined space and wetting behavior. In the application of chemically non-homogeneous surface, its surface properties vary due to a variety of different factors. To improve this problem, it is necessary to use molecular modeling and modern surface techniques, in which make the study of the fluid on chemically non-homogeneous surface be carried out from micro and molecular level, so that the chemical process is optimized to promote the continuous development of chemical industry.
\end{abstract}

\section{Introduction}

As the important impetus for the development of industrial economy, chemistry plays an important role in the economic development of China. In chemical research, mass conservation is the important basis for the study of chemistry, but for the surface of object, the use of this approach cannot measure its quality. This is mainly because the surface of objects are often directly contact with external environment, in this case, after the interaction between the surface of objects and the external environment, it will lead to the surface structure of object to be more complex. Therefore, the previous research methods cannot be solved effectively ${ }^{[1]}$. In chemical engineering, the phenomenon of solid-liquid contact is quite frequently, both applications can effectively improve the efficiency of chemical processes in the process of chemical industry. However, as far the liquid in the hydrophobic and hydrophilic at the same time and the chemically heterogeneity properties of this kind of more complex surface structure as concerned, whether it analyzes from molecular level or limited space, the behavior of wetting and transmission and other chemical processes has certain particularity. Therefore, in the past, the method of surface research for simple hydrophilic or hydrophobic has been completely unable to meet the requirements of current research and the application of chemical industry. In this case, the advantages for the chemically heterogeneity surfaces of fluid attract universal attention, but how to apply this technology in actual chemical engineering has not yet been an effective way at present. The following discussion focuses on chemically non-homogeneous surfaces in the nanoscale. By studying the advantages of this technique in the process of chemical industry, the confined space and wetting behavior of chemically heterogeneous surface are discussed. 


\section{The application of chemically heterogeneous technology in chemical applications}

At present, chemical non-uniform surface plays an important position in the chemical industry of the modification of membrane surface, nano powder and catalyst and so on. When chemical non-homogeneous surface is applied in practical chemical engineering, one end is hydrophilic, and the other end is hydrophobic after modification of surface. This chemical substance is different from single hydrophilic material and hydrophobic material, and it can effectively make water-oil binary system to steadily exist. Because of this feature, the relevant researchers believe that this technology will be able to get an effective promotion and application in heterogeneous catalysis in the future. As the catalyst carrier, its own surface is hydrophilic or hydrophobic, which has direct impact on the catalytic efficiency and adsorption capacity of surface ${ }^{[2]}$. Organic molecules and polar molecules in comparison, organic molecules can easily enter into the hydrophilic hole, or they can absorb the hydrophilic part of catalyst surface, in this case, it will lead to the catalytic reduction of organic reaction activity. In practical study, it is found that the photocatalytic activity of $\mathrm{TiO}^{2}$ can be effectively improved if $\mathrm{TiO}^{2}$ is deposited on the mesoporous silica after hydrophobic modification. In the organic reactions of $\mathrm{H}_{2} \mathrm{O}_{2}$ as oxidant, if hydrophilic or hydrophobic non-uniform titanium silicon molecular sieve is regarded as catalyst after hydrophilic or hydrophobic modification, it can effectively promote the improvement of reaction conversion and selectivity. In the continuous study of this process, we found that the TiSBA15 can improve the ring ethylene oxidation yield of 2.6 times higher than original yield through the silane modification of surface parts. If the surface part of silane is modified as Ti-MCM41, it will be able to improve the ring ethylene oxidation yield of 21 times higher than original yield ${ }^{[3]}$. The reason for this phenomenon is mainly because $\mathrm{H}_{2} \mathrm{O}_{2}$ will be generated $\mathrm{H}_{2} \mathrm{O}$ after reaction. Because the titanium silicon molecular sieve becomes hydrophobic after modification, it won't be polluted by $\mathrm{H}_{2} \mathrm{O}$, in this case, the final catalytic efficiency can get guarantee. It can be seen from the above studies that the rate and the yield of final reaction after chemical non-homogeneous treatment are closely related to the ease of expression and reaction of molecule. To improve the efficiency of catalytic reaction, we need to choose reasonable chemically non-uniform modification.

In the membrane industry, the hydrophilicity and hydrophobicity of membrane surface has great influence on membrane flux, pollution resistance and selectivity. According to the relevant research, it is found that in order to effectively promote the improvement of membrane performance, it is necessary to apply chemically non-homogeneous surface, for example, such as introducing a part of hollow fiber membrane on the surface of sulfur water in polypropylene was hydrophilic allyl propionic acid groups. When the grafting ratio is $3.46 \%$, water contact angle will be reduced from original $120^{\circ}$ to $36^{\circ}$, and it does not appear the situation hydrophobic response. When the grafting ratio is $250 \%$, the membrane water flux will be 10 times higher than that of blank film. If the grafting ratio is $20 \%$, the pure water flux will decline. Related to this problem, the researchers use the sugar surface modification of membrane surface, so that the water molecule selectivity and flux has been effectively improved. And the chemical non-uniform manner is introduced into membrane industry, which can effectively improve the contact area between water molecular and membrane surface so that it can promote the separation efficiency of membrane. From the above 
analysis, it can be seen that the chemically heterogeneous surface of membrane and catalyst have obvious characteristics and advantages in using. But at present, the mechanism has not been studied clearly, and therefore it cannot be purposely applied in chemical industry.

\section{The wettability of chemical non-uniform surface}

Normally, the wettability of cabinet is mainly characterized by contact angle. The wettability of chemically non-homogeneous surfaces can be divided into three types, which are hydrophobic monomolecular layer, hydrophobic material composite surface and rough surface. First, from the nano structure of hydrophobic monomolecular layer formed on the hydrophobic surface, the wettability of the structure is mainly due to the existence of nano bubbles in the hydrophobic surface, while nano bubble itself will affect the non-uniform surface wetting behavior of chemistry. Therefore, in the case of the existence of nano bubbles, it will make the contact of previous two aspects of solid and liquid become three-phase structure, which will affect the wettability of surface. In the chemical industry, the use of nanostructures to influence wettability can be catalyzed and degraded by light, thus it can assure that different nanostructures are present on the same planes, so as to achieve clear change in the wetting gradient and to achieve controllable preparation ${ }^{[4]}$

As far as present situation is concerned, this method is an effective way of chemically inhomogeneous surfaces in chemical application. In addition, the smooth surface of hydrophobic compound is the wetting behavior of chemically non-uniform surface at the micrometer scale, which needs to be explained by the theory of three-phase contact line. The last one is the surface of coarse structure, in which produces two kinds of wetting behaviors, and they are respectively the non-complete contact and complete contact of solid-liquid. The surface tension, rough structure of liquid and the chemical properties of micro structure are the decisive factors influencing the existence of the third phase gas phase on the rough surface. If the contact angle of rough surface increases and lags, it indicates that there is the third phase gas phase, in this case, the actual contact angle of rough surface will also increase as the air fraction increases. Once the air rate reaches certain level, when the contact angle is greater than $150^{\circ}$, it will appear super-hydrophobic performance. Relevant researchers also conduct research on the modification of fluorocarbon coupling agent on the rough aluminum surface. For the case of mixed solutions of water and methanol with different surface tensions, they carry out research on the wettability of flat surfaces and chemically non-uniform surfaces. The study found that if the surface tension of solution is more than $40 \mathrm{mN} \mathrm{m}^{-1}$, the contact angle of flat surface becomes smaller than that of non-uniform surface. And if the tension of solution surface is less than $40 \mathrm{mN}^{\prime} \mathrm{m}^{-1}$, the lyophilic will be increased, and the expected contact angle of flat surface will be greater than that of non-uniform surface. In addition, the wettability of surfactant on PTFE rough surface and the mixed solution between ethanol and water were studied. The study found that when the surface tension of mixed solution between ethanol and water solution constantly changed, the wettability of solution on the surface of PTFE was changed. When the surface tension is less than $27.6 \mathrm{mN} \mathrm{m}^{-1}$, ethanol and water mixed solution can easily enter into the structure of surface micro, and the coating will show lyophilicity in this period. When the surface tension is more than $28 \mathrm{mN} \mathrm{m}^{-1}$, the contact angle of solution on the 
coating layer was more than $120^{\circ}$, and the coating exhibits anti-wetting in this case. Despite the decrease in the absence of surface tension, the surface of coating will exhibit lyophilicity, the air fraction after contacting is always greater than 0.5 , in this state, it is difficult to let solution enter into PTFE rough surface, therefore, there shows lyophobicity between surfactant solution and crude PTFE coating. It can be seen that the wetting behavior of chemically non-homogeneous surface is a king of very complex behavior, and the occurrence of wetting behavior is influenced by many factors, such as the size of surface structure, the nature change after liquid contacting with surface, etc..

\section{The role research of chemically non-uniform surface on molecular transport mechanism}

For the observation of chemically non-uniform surface, in addition to the SEM and STM methods, the molecular modeling technique can effectively realize the understanding of the mechanism of chemical reaction and the chemical non-uniformity of chemically non-uniform surface. Because in actual chemical process, it often cannot simply use the test method to study the problems which exist, for these phenomena are likely to need to occur within the limited micro-channel. In this case, in order to better understand the principle of these limited conditions, you can choose the way of molecular simulation. In the study, it was found that the fluxes of water molecules contained in the biological proteins measured in the experiments were consistent with the fluxes of water molecules in the molecular modeling of microchannels, and because of simulation, the interaction between carbon nanotubes and water molecules exists, so it had a great impact on the flow of water molecules. In addition, the structure of ethanol molecules in carbon nanotubes with different diameters has been studied, and the structure of study indicates that the structure of ethanol was consistent with that of water molecules in this case. In the study, it was also found that the ethanol flux increased in the hydrophobic channel, and it could be seen that the separation of alcohol and water was effectively promoted when the channel was in the hydrophobic environment. In different chemical properties' surface, due to the interaction is different, different fluid molecules will eventually result in different fluid fluxes. After the introduction of some hydroxyl groups into hydrophobic surface, the chemically heterogeneous surface was formed, and then the establishment of nano channel was established, and the flux of water molecules was studied. In the study found that there is the effect of chemically non-uniform surface, and the flow of water molecules in the channel will be severely affected by this. The study found that the hydrophilic groups in aqueous system in confined space under the control of its own scope is less than the range of action, and the residence time of water molecules was affected by the non-polar groups, the distribution of polar group and its size of chemically non-uniform surface. From the point of view of catalytic reaction, the desorption process of products and the adsorption of reactants will be affected. The scale and distribution of chemically heterogeneous surface will also be different in different catalytic reactions. After the hydrophilic carboxyl groups using inner carbon nanotubes to carry out modification, it is regarded as the chemical heterogeneity in confined space, in this environment. It is found that although water molecules has certain distance for the distance of modified groups, it will still be affected, so it can be seen that the effect of chemical modification groups can be 
transferred through the role of fluid molecules, and it is not just the impact of short range in the polar fluid molecules. This characteristic is quite obvious. The modified regions in the carbon nanotubes become the control area in the channel, which seriously affect the transfer of fluid in the channel. Hydrophobic carbon nanotubes were modified by hydrophilic carboxyl groups, on this condition, we need to observe the reaction of water molecules into limited carbon nanotubes. The study found that despite the use of hydrophilic carboxyl groups was modified in the limited channel, but the case of entering carbon nanotubes did not change, even appeared steric effect. Thus, we can see that the water molecules into carbon nanotubes are mainly affected by the chemical effects of non-uniform surface, and the effect of temperature and pressure is relatively small.

\section{Concluding remarks}

In summary, in the continuous development of current chemical industry and social economy, the characteristics of fluid in the chemical heterogeneous surface have attracted the common attention of researchers. But because the wetting behavior of chemically non-uniform surface itself will be subject to the impact of surface geometry, size, chemical properties and fluid properties and so on, in different conditions, the impact of surface on fluid are not the same,

In this study, using molecular simulation technology can realize effectively the deep study for the effect of chemically non-homogeneous surface and fluid. Through clear the mechanism of action, we can achieve better the design of chemically heterogeneous surface, and it can apply this technique in actual chemical industry operation, so as to promote the continuous development of modern chemical industry.

\section{References}

[1] Wang Changsong, Qian Shasha, Lu Xiaohua. The Hydrophilicity / Hydrophobicity of Modified $\mathrm{TiO}^{2}$ Powder and it Adsorption of Methyl Orange [J]. Journal of Nanjing University of Technology (Natural Science Edition), 2014 (3).

[2] Wei Mingjie, Lv Linghong, Zhu Yudan, Guo Xiaojing, Lu Xiaohua. Increasing the Molecular Simulation of the Diffusion of Water Molecules in the Channels of Titanium Dioxide [J]. Journal of Chemical Engineering, 2013 (1).

[3] Ma Ying. Yu Chunmeng. Liu Ke. Cai Xiuqin. Zhang Shaofei. Fang Yu. The Surface Chemical Assembly of Calix [4] Arene and its Application in Gas Sensing Film Creation in Tetrahydrofuran Fluorescence [J]. Imaging Science and Photochemistry, 2015 (1).

[4] Yang Fan, An Qiufeng, Li Xianqi, Wang Kefeng, Wang Qianjin. The Film Morphology and Application Properties of Polyether Block Amino Silicon BPEAS [J]. Textile journal, 2011 (7). 